\title{
Strictly Linear Light Cones in Long-Range Interacting Systems of Arbitrary Dimensions
}

\author{
Tomotaka Kuwahara $\odot^{*}$ \\ Mathematical Science Team, RIKEN Center for Advanced Intelligence Project (AIP), \\ 1-4-1 Nihonbashi, Chuo-ku, Tokyo 103-0027, Japan \\ and Interdisciplinary Theoretical and Mathematical Sciences Program (iTHEMS), \\ RIKEN 2-1, Hirosawa, Wako, Saitama 351-0198, Japan
}

\author{
Keiji Saito \\ Department of Physics, Keio University, Yokohama 223-8522, Japan
}

(Received 25 March 2020; revised 18 June 2020; accepted 19 June 2020; published 13 July 2020)

\begin{abstract}
In locally interacting quantum many-body systems, the velocity of information propagation is finitely bounded, and a linear light cone can be defined. Outside the light cone, the amount of information rapidly decays with distance. When systems have long-range interactions, it is highly nontrivial whether such a linear light cone exists. Herein, we consider generic long-range interacting systems with decaying interactions, such as $R^{-\alpha}$ with distance $R$. We prove the existence of the linear light cone for $\alpha>2 D+1$ ( $D$, the spatial dimension), where we obtain the Lieb-Robinson bound as $\left\|\left[O_{i}(t), O_{j}\right]\right\| \lesssim t^{2 D+1}(R-\bar{v} t)^{-\alpha}$ with $\bar{v}=\mathcal{O}(1)$ for two arbitrary operators $O_{i}$ and $O_{j}$ separated by a distance $R$. Moreover, we provide an explicit quantum-state transfer protocol that achieves the above bound up to a constant coefficient and violates the linear light cone for $\alpha<2 D+1$. In the regime of $\alpha>2 D+1$, our result characterizes the best general constraints on the information spreading.
\end{abstract}

DOI: 10.1103/PhysRevX.10.031010

\section{INTRODUCTION}

In deep understanding of many-body physics, we necessarily encounter the question on how fast information propagates in the dynamics. In this context, the most fundamental principle is the causality; that is, in relativistic systems, information propagation is completely prohibited outside the light cone. On the other hand, in nonrelativistic quantum many-body systems, a rigorous light cone is not defined. Lieb and Robinson proved in 1972 [1] that an effective light cone can be defined, outside which information propagation exponentially decreases with distance. In their study, the effective light cone is characterized by the so-called Lieb-Robinson velocity.

The Lieb-Robinson bound imposes one of the most fundamental restrictions to the dynamics [2-13] and has been improved in various ways [9,14-20]. Moreover, after Hastings' work on the Lieb-Schultz-Mattis theorem [21], the Lieb-Robinson bound has been recognized as a crucial

\footnotetext{
*tomotaka.kuwahara@ riken.jp

†saitoh@rk.phys.keio.ac.jp
}

Published by the American Physical Society under the terms of the Creative Commons Attribution 4.0 International license. Further distribution of this work must maintain attribution to the author(s) and the published article's title, journal citation, and DOI.
Subject Areas: Quantum Information

ingredient for analyzing the universal physics in manybody systems, such as quasiadiabatic continuation [22], the area law of entanglement [23-26], thermalization [27-30], quantization of Hall conductance [31,32], stability of topological order [33-35], the clustering theorem for correlation functions [14,15,36-38], the effective Hamiltonian theory [39,40], and classical simulation of many-body systems [41-47]. More recently, the LiebRobinson bound has been further applied to the digital quantum simulation of many-body systems [48-50] and quantum information scrambling [51-63], where the LiebRobinson velocity gives an upper bound of butterfly speed [51]. In addition, experimental advancement enables direct observation of the Lieb-Robinson bounds [64-70].

In the case of short-range interacting spin systems, an effective light cone is characterized by a finite velocity, and information propagation is restricted inside the "linear light cone." However, when we consider long-range interacting systems, the existence of a linear light cone is quite subtle, because long-range interactions enable immediate communication between two arbitrarily distant parties. Here, longrange interaction implies that the interaction strength between separated sites shows a power-law decay of $R^{-\alpha}$ with the distance $R$. Depending on the exponent $\alpha$, both the linear and nonlinear light cones can appear. Recent experiments have realized long-range interacting systems with various values of $\alpha$ [71-80], and, hence, exploring the 
universal aspects of long-range interacting systems is attracting increasing attention [81-87]. From these backgrounds, one of the most important and intriguing open problems is the so-called linear light cone problem, which clarifies whether linear right cones can exist in long-range interacting systems, and what the general criterion is for it.

So far, various studies have clarified dynamical properties in the long-range interacting systems [88-104]. As one of the generic aspects of the Lieb-Robinson bound, Refs. [15,17,105-107] show that the amount of information propagation is suppressed at least outside the effective light cone exponentially growing in time, irrespective of $\alpha$. Later, a more detailed universal upper bound was provided by Foss-Feig et al. [108]. They prove that the effective light cone is at most polynomial with respect to time; in more detail, the shape of the light cone is given by $t^{(\alpha-D+1) /(\alpha-2 D)}$ $(\alpha>2 D)$ with $D$ as the spatial dimensions. However, despite significant efforts [49,109-111], the critical value of $\alpha$ to obtain a linear light cone is still unclear even at the numerical level.

In this work, we rigorously prove that a linear light cone is obtained in generic long-range interacting systems under the condition of $\alpha>2 D+1$. As related work, in onedimensional two-body interacting systems, the long-range Lieb-Robinson bound has been proved very recently in the form of $\left\|\left[O_{i}(t), O_{j}\right]\right\| \lesssim t / R$ for $\alpha>3$ [112], which gives a nontrivial upper bound up to the time $t=\mathcal{O}(R)$. In our analyses, the Hamiltonian is not restricted to few-body interactions and is applicable to arbitrary spatial dimensions. Our Lieb-Robinson bound is given in the stronger form of $\left\|\left[O_{i}(t), O_{j}\right]\right\| \lesssim t^{2 D+1}(R-\bar{v} t)^{-\alpha}$. However, only the above commutation relation is not sufficient to upper bound the entire information propagation outside the light cone. To obtain a linear light cone in a strict sense [see Eq. (5) and Fig. 1], we also prove that the error of the local approximation of $O_{i}(t)$ decays as $t^{D+1} R^{-\alpha+D}$ outside the

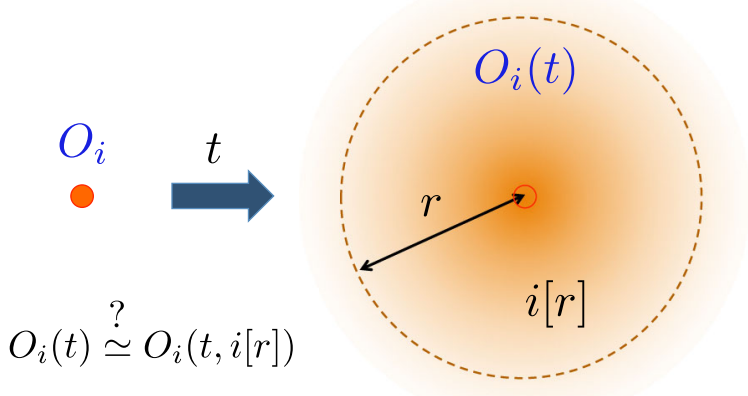

FIG. 1. When we consider time evolution $O_{i}(t)$ of a local operator $O_{i}$, the quasilocality of the interaction ensures that $O_{i}(t)$ is well approximated by an operator defined on a ball region $i[r]$ having the maximum distance of $r$ from $i$. The dynamics define the linear light cone if we can achieve an arbitrary approximation error for $r=\mathcal{O}(t)$ as in Eq. (5). light cone [see Eq. (7) below]. Our result can improve various existing analyses that depend on a polynomial light cone $[49,108]$.

We also discuss whether $\alpha=2 D+1$ is the critical value to ensure a linear light cone in general setups. In other words, we investigate the achievability of our LiebRobinson bound and the possibility to violate the linear light cone for $\alpha<2 D+1$, by which we show that our bound is the best general upper bound. We consider a quantum-state transfer protocol through the dynamics on a spin network and give an explicit example that achieves our Lieb-Robinson bound for $\alpha>2 D+1$ and violates the linear light cone for $\alpha<2 D+1$. Our protocol is applied to (1/2)-spin systems and comprises only the Ising-type longrange interactions and simple short-range interactions that generate the controlled NOT gate operation. This example ensures the optimality of our results.

The rest of this paper is organized as follows. In Sec. II, we formulate the precise setting and state the main results and their implications. In Sec. III, we sketch the intuitive explanation for the condition that a linear light cone appears. We also show the brief strategy for the proof. In Sec. IV, we discuss the optimality of the present LiebRobinson bound. In more detail, we explicitly show a quantum-state transfer protocol which achieves our theoretical upper bound up to a coefficient. Finally, in Sec. V, we summarize the paper, making a brief discussion.

\section{MAIN RESULTS}

We consider a quantum many-body system with $n$ sites, where each site is located on a $D$-dimensional lattice with the total set $\Lambda(|\Lambda|=n)$. We assume that a finitedimensional Hilbert space is assigned to each of the sites.

For simplicity, we restrict ourselves to two-body interactions, but our results are extended to a more general setup, as shown in the Appendix A. We focus on the following Hamiltonian $H$ with power-law decaying interactions:

$$
H=\sum_{i, j \in \Lambda} h_{i, j}+\sum_{i=1}^{n} h_{i} \quad \text { with } \quad\left\|h_{i, j}\right\| \leq \frac{g_{0}}{d_{i, j}^{\alpha}}
$$

where $d_{i, j}$ is the distance between the sites $i$ and $j$, namely, the minimum path length from the site $i$ to $j,\left\{h_{i, j}\right\}_{i<j}$ are the bipartite interaction operators, $\left\{h_{i}\right\}_{i=1}^{n}$ are the local potentials, $g_{0}$ is a positive constant of $\mathcal{O}(1)$, and $\|\cdots\|$ is the operator norm. Although in some literature [113,114] the long-range interaction often implies the power-law decaying interactions with $\alpha \leq D$, we refer to systems with arbitrary power-law decaying interactions as longrange interacting systems in distinction from the finiterange (or exponentially decaying) interactions.

Our analysis can be also applied to a time-dependent Hamiltonian, but, for simplicity, we present the analysis 
only for the time-independent case. One typical example is the following one-dimensional long-range Ising model:

$$
H=\sum_{i<j} \frac{g_{0}}{d_{i, j}^{\alpha}} \sigma_{i}^{x} \sigma_{j}^{x}+B \sum_{i} \sigma_{i}^{z} \quad(D=1),
$$

where $\left\{\sigma^{x}, \sigma^{y}, \sigma^{z}\right\}$ are the Pauli matrices. This class of Hamiltonians is experimentally realized for the power-law exponent in $\alpha \leq 3$ [69,79].

We are now interested in the time evolution using the Hamiltonian $H$. For simplicity, we consider an operator $O_{i}$ that is locally defined on the site $i$ and analyze

$$
O_{i}(t):=e^{i H t} O_{i} e^{-i H t} .
$$

Mainly, we focus on the following two quantities:

$$
\left\|\left[O_{i}(t), O_{j}\right]\right\| \quad \forall j \in \Lambda
$$

and

$$
\begin{aligned}
& \left\|\left[O_{i}(t)-O_{i}(t, i[r])\right]\right\| \text { with } \\
& O_{i}(t, i[r]):=\frac{1}{\operatorname{tr}_{i[r]^{c}}(\hat{1})} \operatorname{tr}_{i[r]^{c}}\left[O_{i}(t)\right] \otimes \hat{1}_{i[r]^{c}},
\end{aligned}
$$

where $i[r]$ denotes the set of sites having a maximum distance of $r$ from the site $i$ and $i[r]^{c}$ is its complement set. The quantity (4) characterizes the error of the local approximation for the time-evolved operator $O_{i}(t)$ into the region $i[r]$ (see Fig. 1). We note that the decay of Eq. (4) is not necessarily derived only from the decay of Eq. (3).

Here, we define the linear light cone in the following sense. We say that the Hamiltonian dynamics $e^{-i H t}$ has a linear light cone if the following inequality holds for an arbitrary error $\delta \in \mathbb{R}$ and $t$ :

$$
\left\|\left[O_{i}(t)-O_{i}(t, i[r])\right]\right\| \leq \delta \quad \text { for } r \geq v_{t, \delta}|t|,
$$

where $v_{t, \delta}$ decreases in time and eventually converges to a finite value (i.e., $v_{\infty, \delta}=$ const). From the definition, the amount of information propagation is smaller than $\delta$ outside the region separated by the distance $v_{t, \delta}|t|$.

Here, we show our main results. For $\alpha>2 D+1$, the Hamiltonian in Eq. (1) satisfies the Lieb-Robinson bound for Eq. (3) as

$$
\left\|\left[O_{i}(t), O_{j}\right]\right\| \leq \mathcal{C}_{H}|t|^{2 D+1}(R-\bar{v}|t|)^{-\alpha}
$$

with $R=d_{i, j}$. In addition, the Lieb-Robinson bound for Eq. (4) is given by

$$
\left\|\left[O_{i}(t)-O_{i}(t, i[R])\right]\right\| \leq \mathcal{C}_{H}^{\prime}|t|^{D+1}(R-\bar{v}|t|)^{-\alpha+D},
$$

where $R>\bar{v}|t|$ is considered and $\mathcal{C}_{H}, \mathcal{C}_{H}^{\prime}$, and $\bar{v}$ are constants that depend only on the parameters $\left\{D, g_{0}, \alpha\right\}$ and a geometric constant defined by the lattice structure. We emphasize that the same upper bound is obtained for generic operators $O_{X}$ and $O_{Y}$ (see Appendix A).

As long as we consider the commutator for local observables, the first inequality (6) is stronger than the second one (7) in the sense that asymptotic decay is as small as $\mathcal{O}\left(R^{-\alpha}\right)$. From the inequality (7), we can calculate the Lieb-Robinson velocity $v_{\delta, t}$ defined in Eq. (5):

$$
v_{\delta, t}=\bar{v}+c \delta^{-[1 /(\alpha-D)]}|t|^{(-\alpha+2 D+1) /(\alpha-D)} \stackrel{t \rightarrow \infty}{\longrightarrow} \bar{v},
$$

where $c$ is a constant of $\mathcal{O}(1)$ and where we use the condition $\alpha>2 D+1$.

\section{INTUITIVE EXPLANATION OF THE CONDITION $\alpha>2 D+1$}

We here show an intuitive explanation of why the condition $\alpha>2 D+1$ appears. The point to obtain the linear light cone is that the contribution to the LiebRobinson velocity from the long-range interaction with very large distance becomes asymptotically negligible. To get better insights into this condition, we consider the simplest setup as follows.

Let us consider the Hamiltonian given by $\mathrm{H}=\mathrm{H}_{1}+\mathrm{H}_{2}$, where $H_{1}$ has only nearest-neighbor interactions, while $H_{2}$ consists of the long-range interactions with the length scales from $\ell$ to $2 \ell$ as $H_{2}=\sum_{\ell \leq d_{i, j} \leq 2 \ell} h_{i, j}$. Note that the condition (1) implies $\left\|h_{i, j}\right\| \leq g_{0} d_{i, j}^{-\alpha}$. We eventually take the large $\ell$ limit to consider asymptotic behavior arising from the interactions of the large distance. Note that the Hamiltonian $H_{1}$ consists of short-range interactions; hence, the unitary operator $e^{-i H_{1} t}$ satisfies the standard Lieb-Robinson bound $[1,14,15]$ giving a finite LiebRobinson velocity. Here, we denote it by $v_{1}$. We focus on the time range of $t \lesssim \ell / v_{1}$ and then consider the condition for which the Lieb-Robinson velocity for the Hamiltonian $H$ is given by a finite velocity related to $v_{1}$ in the large $\ell$ limit.

As a simple exercise, we first consider the product of the unitary operators $e^{-i H_{1} t} e^{-i H_{2} t}$. Then, to estimate the contribution from the long-range interactions, we have only to consider the Lieb-Robinson bound for $e^{-i H_{2} t}$. The LiebRobinson bound for $H_{2}$ is given by $e^{-c\left(x / \ell-v_{\ell} t\right)}$, and $v_{\ell}$ is proportional to the one-site energy:

$$
g=\max _{i \in \Lambda} \sum_{j \in \Lambda}\left\|h_{i, j}\right\|=\mathcal{O}\left(\ell^{-\alpha+D}\right),
$$

where $\sum_{j \in \Lambda}\left\|h_{i, j}\right\| \leq g_{0} \sum_{j: \ell \leq d_{i, j} \leq 2 \ell} d_{i, j}^{-\alpha}$ is a summation of all the interaction terms that act on the site $i$. Hence, the Lieb-Robinson velocity is proportional to $\ell^{-\alpha+D+1}$, which vanishes in the limit of $\ell \rightarrow \infty$ for $\alpha>D+1$. Therefore, the unitary operator $e^{-i H_{1} t} e^{-i H_{2} t}$ has finite Lieb-Robinson velocity $v_{1}$ as long as $\alpha>D+1$. 


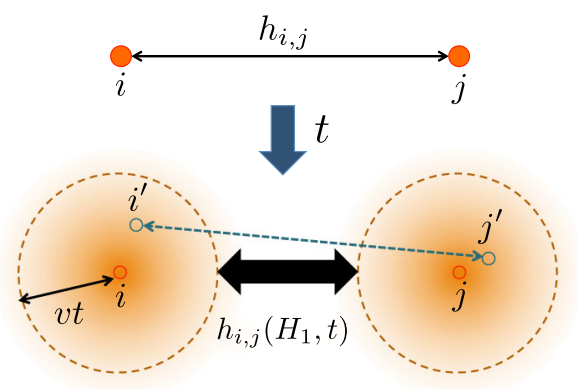

FIG. 2. Effect of the operator spreading. When a bipartite interaction $h_{i, j}$ evolves using the Hamiltonian $H_{1}$, interactions between the sites $i^{\prime}$ and $j^{\prime}$ such that $d_{i, i^{\prime}} \lesssim v t$ and $d_{j, j^{\prime}} \lesssim v t$ are effectively induced. The operator $h_{i, j}\left(H_{1}, t\right)$ now acts on a subsystem with roughly $\mathcal{O}\left(t^{D}\right)$ sites. Hence, one-site energy of $H_{2}\left(H_{1}, t\right)$ defined as in Eq. (9) becomes $\mathcal{O}\left(t^{D}\right)$ times the original one.

Now, let us discuss the unitary operator $e^{-i\left(H_{1}+H_{2}\right) t}$. For this unitary operator, we use the following representation to decompose the contributions from $\mathrm{H}_{1}$ and $\mathrm{H}_{2}$ :

$$
e^{-i\left(H_{1}+H_{2}\right) t}=e^{-i H_{1} t} \mathcal{T} e^{-i \int_{0}^{t} H_{2}\left(H_{1}, \tau\right) d \tau},
$$

where $H_{2}\left(H_{1}, \tau\right):=e^{i H_{1} \tau} H_{2} e^{-i H_{1} \tau}$ and $\mathcal{T}$ denotes the timeordering operator. Because the one-site operator spreads up to a distance $\mathcal{O}(\tau)$ owing to the time evolution $e^{-i H_{1} \tau}$ (Fig. 2), the one-site energy is now given by

$$
g(\tau)=\max _{i \in \Lambda} \sum_{Z: Z \ni i}\left\|h_{\tau, Z}\right\|=\tau^{D} \mathcal{O}\left(\ell^{-\alpha+D}\right)
$$

where $h_{\tau, Z}$ is an interaction term on the subset $Z$ that constitutes $H_{2}\left(H_{1}, \tau\right)$, i.e., $H_{2}\left(H_{1}, \tau\right)=\sum_{Z} h_{\tau, Z}$. Therefore, the time evolution $\mathcal{T} e^{-i \int_{0}^{t} H_{2}\left(H_{1}, \tau\right) d \tau}$ gives the LiebRobinson bound as $e^{-c\left(x / \ell-v_{t, \ell} t\right)}$ with the velocity $v_{t, \ell} \propto$ $g(t)=t^{D} \mathcal{O}\left(\ell^{-\alpha+D}\right)$. For $t \lesssim \ell / v_{1}$, this estimation provides the Lieb-Robinson velocity as $t^{D} l^{-\alpha+D+1} \lesssim \mathcal{O}\left(\ell^{-\alpha+2 D+1}\right)$. Hence, the contribution to the Lieb-Robinson velocity from $\mathrm{H}_{2}$ vanishes in the limit of $\ell \rightarrow \infty$ for $\alpha>2 D+1$. This result leads to the finite Lieb-Robinson velocity $v_{1}$ for the unitary operator $e^{-i\left(H_{1}+H_{2}\right) t}$.

In summary, the spread of the operator changes the effective one-site energy by $t^{D}$ times [see Eq. (9)], which yields the condition of $\alpha>2 D+1$ for the linear light cone. In our proof for the general Hamiltonian (1), we decompose the total length scale into pieces and consider the multiunitary decomposition by generalizing Eq. (8) (see also Appendix B). We then obtain the Lieb-Robinson bound for each of the decomposed unitary operators and connect them into a single Lieb-Robinson bound. The technical difficulties lie in that we need to connect infinitely many Lieb-Robinson bounds; in the step-by-step connections, a simple estimation makes the Lieb-Robinson velocity diverge rapidly, and, hence, highly refined analyses are required to obtain a finite velocity.

\section{OPTIMALITY OF THE PRESENT LIEB-ROBINSON BOUND}

We have proved that the condition $\alpha>2 D+1$ is a sufficient condition for arbitrary Hamiltonians to have the linear light cone. We here discuss whether this condition can be further improved. It has been conjectured in previous studies $[49,94]$ that the best general condition may be given by $\alpha>D+1$ from numerical and theoretical analyses of specific models. Against the conventional expectation, we show that any improvement from $\alpha>$ $2 D+1$ is impossible as long as we consider the general long-range interacting systems (1). In the following, we explicitly provide a quantum-state transfer protocol that achieves a nonlinear light cone for $\alpha<2 D+1$.

We follow a similar setup as in Ref. [2] and consider the quantum-state transfer between two separated spins $A$ and $B$ through a spin network, where we define $R$ as the distance between the spins $A$ and $B$ (Fig. 3). We start from the initial state $|\psi\rangle$ such that all spins are given by $|0\rangle$, namely, $|\psi\rangle:=|0\rangle^{\otimes n}$. We then apply the unitary operation $U_{0}$ or $U_{1}$ to the spin $A$, where $U_{0}$ is the identity operator

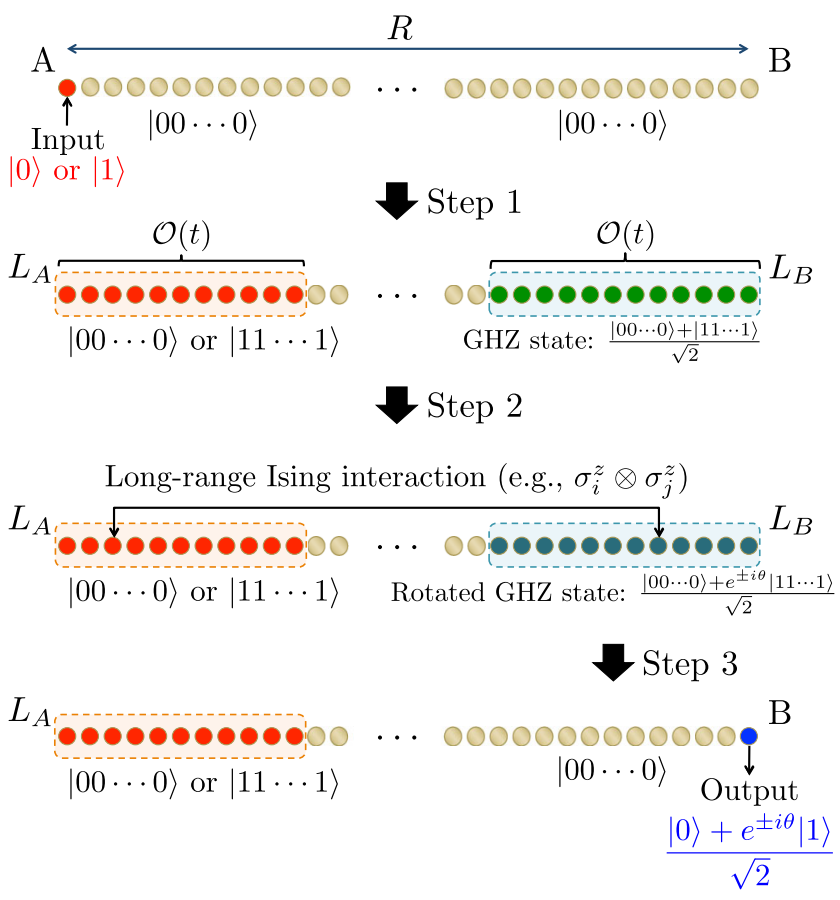

FIG. 3. Schematic of our state-transfer protocol. We show many copies of the input state here and transform the receiver into the GHZ state. The protocol effectively amplifies the long-range interaction strength between the sender and the receiver by $t^{2 D}$ times $(D=1)$. We can see that this simple protocol gives an example that realizes the intuitive picture in Fig. 2. We here consider 1D systems, but the generalization to high-dimensional systems is straightforward. 
and $U_{1}$ is the spin flip operator (i.e., $U_{1}=|0\rangle\langle 1|+| 1\rangle\langle 0|$ ). After applying $U_{0}$ or $U_{1}$, the initial quantum state is transformed into $\left|\psi_{s}\right\rangle=|s\rangle \otimes|0\rangle^{\otimes n-1}$ with $s=0,1$. After time $t$, the quantum state evolves according to the unitary time operation $U(t)=\mathcal{T}\left[e^{-i \int_{0}^{t} H(\tau) d \tau}\right]$. We then define the output state for spin $B$ as

$$
\rho_{B}^{(s)}:=\operatorname{tr}_{B^{c}}\left[U(t)\left|\psi_{s}\right\rangle\left\langle\psi_{s}\right| U(t)^{\dagger}\right] .
$$

If we can distinguish between the state $\rho_{B}^{(0)}$ from $\rho_{B}^{(1)}$ with the probability 1 , we can achieve the perfect quantum-state transfer.

By utilizing the Lieb-Robinson bound (6), we have [2]

$$
\left\|\rho_{B}^{(1)}-\rho_{B}^{(0)}\right\|_{1} \lesssim|t|^{2 D+1} R^{-\alpha}
$$

where $\|\cdot\|_{1}$ is the trace norm. When $\left\|\rho_{B}^{(1)}-\rho_{B}^{(0)}\right\|_{1}=2$, the two states $\rho_{B}^{(0)}$ and $\rho_{B}^{(1)}$ are orthogonal to each other and completely distinguishable. We prove that the upper bound of Eq. (11) is achievable by combining controlled-NOT-type short-range interactions and the Ising-type long-range interactions.

We here consider a one-dimensional (1/2)-spin system and decompose the time evolution into three steps (see Fig. 3). In each of the steps, we take a time of $\mathcal{O}(t)$ (e.g., $t / 3)$. In the first step, we copy the state of the spin $A$ using the controlled NOT (CNOT) gate operation, which is generated by a simple bipartite interaction [94]. We define the subset $L_{A}$ as the spins that are the same state as that of spin $A$ (see Fig. 3 ). We consider the dynamics by short-range interactions, and the number of spins in the subset $L_{A}$ is thus upper bounded by $\mathcal{O}(t)$. At the same time, we prepare the Greenberger-Horne-Zeilinger (GHZ) state that includes the spin $B$. The GHZ state is also generated by combining the rotation of the spin $B$ and the CNOT gate operation. We define the subset $L_{B}$ as the spins that are involved in the GHZ state. Owing to the short-rangeness of the interactions, the number of spins in $L_{B}$ is also of $\mathcal{O}(t)$.

In the second step, we apply long-range Ising interactions by the Hamiltonian

$$
H_{\text {Ising }}=\frac{g}{R^{\alpha}} \sum_{i \in L_{A}} \sum_{j \in L_{B}} \sigma_{i}^{z} \otimes \sigma_{j}^{z}
$$

with $g \leq g_{0}$. Because the states of spins $L_{A}$ are given by $|0 \ldots 0\rangle$ or $|1 \ldots 1\rangle$, the unitary time evolution by $H_{\text {Ising }}$ changes only the phase factor of the GHZ state of spins $L_{B}$; that is, the GHZ state $(|00 \ldots 0\rangle+|11 \ldots 1\rangle) / \sqrt{2}$ is rotated as $\left(|00 \ldots 0\rangle+e^{ \pm 2 i \theta}|11 \ldots 1\rangle\right) / \sqrt{2}$ with \pm depending on the states of spins $L_{A}$. Simple and straightforward calculations can give the phase shift $\theta$ as $\theta=g \mathcal{O}(t)\left|L_{A}\right| \cdot\left|L_{B}\right| R^{-\alpha}$. Then, if $\theta$ is taken as $\theta=\pi / 4$, the rotated GHZ states $\left(|00 \ldots 0\rangle+e^{ \pm 2 i \theta}|11 \ldots 1\rangle\right) / \sqrt{2}$ are mutually orthogonal.
In the final step, we untangle the rotated GHZ state and concentrate the phase term on the spin $B$, which transforms this state to $|00 \ldots 0\rangle \otimes\left(|0\rangle+e^{ \pm 2 i \theta}|1\rangle\right) / \sqrt{2}$. This procedure is also performed by the CNOT gate operation; hence, we need only the short-range interactions. In this protocol, we obtain the lower bound of

$$
\left\|\rho_{B}^{(1)}-\rho_{B}^{(0)}\right\|_{1}=2 \sin (2 \theta) \gtrsim t\left|L_{A}\right| \cdot\left|L_{B}\right| R^{-\alpha} .
$$

Considering that $\left|L_{A}\right|=\left|L_{B}\right|=\mathcal{O}(t)$, we can achieve the theoretical upper bound of Eq. (6) with $D=1$. Thus, as long as $\alpha<3$, the information can reach a distance of $R=\mathcal{O}\left(t^{3 / \alpha}\right)$. This protocol can be generalized to highdimensional setups, and we obtain the same lower bound as Eq. (13), where we have $\left|L_{A}\right|=\left|L_{B}\right|=\mathcal{O}\left(t^{D}\right)$. Then, the shape of the light cone becomes $t^{(2 D+1) / \alpha}$.

This simple quantum model [i.e., two-body interaction and (1/2)-spin systems] already saturates the LiebRobinson bound of Eq. (6); hence, our condition $\alpha>$ $2 D+1$ for the linear light cone cannot be improved unless we consider a special class of Hamiltonians. Recently, a similar protocol to achieve the Lieb-Robinson bound (6) was given [115] with a more explicit lower bound on the commutator $\left\|\left[O_{i}(t), O_{j}\right]\right\|$.

\section{SUMMARY AND DISCUSSION}

In this study, we prove the existence of the linear light cone [see Eq. (5) for the definition] in general long-range interacting systems, where the interaction decays as according to the relation $R^{-\alpha}(\alpha>2 D+1)$ with respect to the distance $R$. Our Lieb-Robinson bound in (6) provides an approximate commutation relation as $\left\|\left[O_{i}(t), O_{j}\right]\right\| \lesssim$ $t^{2 D+1}(R-\bar{v} t)^{-\alpha}$, with rapid decay beyond $r \gtrsim \bar{v} t$. Moreover, the error of the local approximation for $O_{i}(t)$ is estimated as in Eq. (7), namely, $\left\|O_{i}(t)-O_{i}(t, i[R])\right\| \lesssim$ $t^{D+1}(R-\bar{v} t)^{-\alpha+D}$. Our result is obtained for Hamiltonians with two-body interactions but can be extended to a more general setup [see Eqs. (A1) with (A2) in Appendix A], where even the few-body interactions are not assumed. We also show an explicit example that our Lieb-Robinson bound is saturated for $\alpha>2 D+1$, and the linearity of the light cone deteriorates for $\alpha<2 D+1$. Therefore, our condition for the linear light cone is optimal as long as we consider the general class of Hamiltonians. Although we consider Hamiltonian dynamics throughout this work, we expect that the same analysis can be applied to generic Markovian dynamics using the procedures in Refs. [18,116].

We finally present an open question. In the present work, although we provide the optimal Lieb-Robinson bound for $\alpha>2 D+1$, it is still unclear what can be obtained in the $\alpha \leq 2 D+1$ regimes. The most important problem is to identify the regime of the exponent $\alpha$ that ensures the polynomial (or superlinear) light cone. A state-of-the-art 
analysis [49] has defined the polynomial light cone in the form $r=t^{(\alpha-D) /(\alpha-2 D)}$ for $\alpha>2 D$. In contrast, the superpolynomial light cone has been explicitly shown for $\alpha \leq D$ [94]. In tackling this problem, the simplest case with only two length scales as in Eq. (8) may be a good starting point. We expect that our present analysis will provide a better polynomial light cone for high-dimensional systems.

\section{ACKNOWLEDGMENTS}

T. K. was supported by the RIKEN Center for AIP and JSPS KAKENHI Grant No. 18K13475. K. S. was supported by JSPS Grants-in-Aid for Scientific Research (Grants No. JP16H02211, No. JP19H05791, and No. JP19H05603).

\section{APPENDIX A: FORMAL EXPRESSION OF THE THEOREM}

We demonstrate our theorem here in a general manner. First, some necessary notations are provided; for arbitrary subsystems $X, Y \subset \Lambda$, we define $d_{X, Y}$ as the shortest path length on the lattice that connects $X$ and $Y$. If $X \cap Y \neq \varnothing$, $d_{X, Y}=0$. For a subset $X \subseteq \Lambda$, we define $\operatorname{diam}(X):=$ $\max _{i, j \in X}\left(d_{i, j}\right)+1$, the cardinality $|X|$ as the number of vertices contained in $X$, and the complementary subset of $X$ as $X^{c}:=\Lambda \backslash X$.

We consider a general class of the Hamiltonian beyond the two-body interaction (1) as

$$
H=\sum_{Z \subseteq \Lambda} h_{Z},
$$

where each of the interaction terms $\left\{h_{Z}\right\}_{Z \subseteq \Lambda}$ acts on the sites in $Z \subseteq \Lambda$. Notably, we do not assume the few-body interaction here; i.e., $|Z|$ can be arbitrarily large up to $|Z|=n$. Therefore, the Hamiltonian $H$ includes macroscopic interactions such as $\sigma_{1}^{z} \otimes \sigma_{2}^{z} \otimes \cdots \otimes \sigma_{n}^{z}$. However, the assumption (A2) below restricts the amplitude of such interactions as poly $(1 / n)$. Here, the only assumption is the following power-law decay of the interactions:

$$
\begin{aligned}
& \sup _{i \in \Lambda} \sum_{Z: Z \ni i, \operatorname{diam}(Z) \geq r}\left\|h_{Z}\right\| \leq g r^{-\alpha+D}, \\
& \sup _{i, j \in \Lambda} \sum_{Z: Z \ni\{i, j\}}\left\|h_{Z}\right\| \leq g_{0}\left(d_{i, j}+1\right)^{-\alpha}
\end{aligned}
$$

with

$$
\alpha>2 D+1
$$

for an arbitrary site pair of $\{i, j\} \subset \Lambda$. Here, $\sum_{Z: Z \ni\{i, j\}}$ denotes the summation that encompasses all the interaction terms $\left\{h_{Z}\right\}_{Z \subseteq \Lambda}$, including the sites $i$ and $j$, and $\|\cdots\|$ is the operator norm. By considering an appropriate energy unit, we set $g=1$.

To formulate our main theorem, we first define the coarse-grained subsets (see Fig. 4). For a subset $X \subseteq \Lambda$, we first define $X[r]$ as the extended subset

$$
X[r]:=\left\{i \in \Lambda \mid d_{X, i} \leq r\right\},
$$

where $X[0]=X$ and $r$ is an arbitrary positive number. We also define the coarse-grained total set $\Lambda^{(\xi)}$ as the minimum subset such that $\Lambda^{(\xi)}[\xi]=\Lambda$, namely,

$$
\Lambda^{(\xi)}:=\arg \min _{Z \subseteq \Lambda \mid Z[\xi]=\Lambda}|Z|,
$$

where $\Lambda^{(0)}=\Lambda$. Similarly, for an arbitrary subset $X \subseteq \Lambda$, we define $X^{(\xi)} \subseteq \Lambda^{(\xi)}$ as follows:

$$
X^{(\xi)}:=\arg \min _{Z \subseteq \Lambda^{(\xi)} \mid Z[\xi] \supset X}|Z|,
$$

where $X^{(0)}=X$. From the definition, the cardinality of the subset $X^{(\xi)}$ is roughly $(1 / \xi)^{D}$ times that of the original, namely, $\left|X^{(\xi)}\right| \approx(1 / \xi)^{D}|X|$.

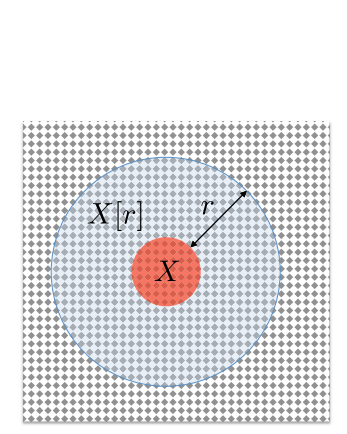

(a) Definition of $X[r]$

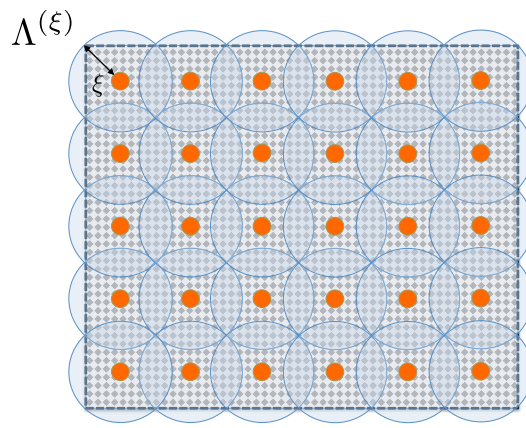

(b) Definition of $\Lambda^{(\xi)}$

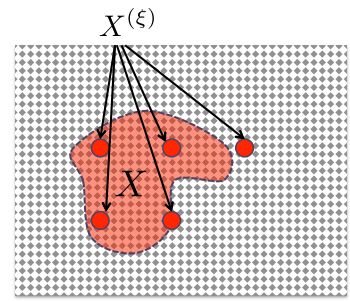

(c) Definitions of $X^{(\xi)}$

FIG. 4. (a) Extended subset. The subset $X[r]$ is defined by extending the original subset $X$ by a distance $r$. (b) The subset $\Lambda^{(\xi)} \subseteq \Lambda$ is the coarse-grained lattice (orange sites) which is defined as the minimum subset such that $\Lambda^{(\xi)}[\xi]=\Lambda$. (c) For an arbitrary subset $X \subseteq \Lambda$ (pink region), $X^{(\xi)} \subseteq \Lambda^{(\xi)}$ is the coarse-grained subset (red sites), which is defined as the minimum subset such that $X^{(\xi)}[\xi] \supseteq X$. The cardinality of the subset $X^{(\xi)}$ is roughly $(1 / \xi)^{D}$ times as the original one, namely, $\left|X^{(\xi)}\right| \approx(1 / \xi)^{D}|X|$. 
Using the notation $X^{(\xi)}$, we state our main theorem as follows.

Main theorem.-Let us consider the long-range interacting Hamiltonian $H$ of the form (A1) with the assumption (A2). For $|t| \geq 1$, this Hamiltonian $H$ satisfies the LiebRobinson bound for arbitrary operators $O_{X}$ and $O_{Y}$ that are supported on $X \subseteq \Lambda$ and $Y \subseteq \Lambda$, respectively:

$$
\frac{\left\|\left[O_{X}(t), O_{Y}\right]\right\|}{\left\|O_{X}\right\| \cdot\left\|O_{Y}\right\|} \leq \mathcal{C}_{H}\left|X^{(\bar{v}|t|)}\right| \cdot\left|Y^{(\bar{v}|t|)}\right| \frac{|t|^{2 D+1} \log ^{2 D}(x+1)}{(x-\bar{v}|t|)^{\alpha}}
$$

and

$$
\frac{\left\|\left[O_{X}(t), O_{Y}\right]\right\|}{\left\|O_{X}\right\| \cdot\left\|O_{Y}\right\|} \leq \mathcal{C}_{H}^{\prime}\left|X^{(\bar{v}|t|)}\right|^{2} \frac{|t|^{D+1} \log ^{2 D}(x+1)}{(x-\bar{v}|t|)^{\alpha-D}},
$$

where $\mathcal{C}_{H}, \mathcal{C}_{H}^{\prime}$, and $\bar{v}$ are constants that depend only on parameters $\left\{D, g_{0}, \alpha\right\}$ and a geometric constant that is determined by the lattice structure. Note that we use the notation from Eq. (A6) with $\xi=\bar{v}|t|$.

Here, the coefficient $\log ^{2 D}(x+1)$ exists because of a technical reason, which results from the macroscopic interactions in Eq. (A1) [e.g., $\mathcal{O}(n)$-body interactions]. If we restrict ourselves to the few-body (or $k$-local) Hamiltonians with $k=\mathcal{O}(1)$, namely,

$$
H=\sum_{Z \subseteq \Lambda,|Z| \leq k} h_{Z}
$$

the Lieb-Robinson bound is slightly improved as follows.

Main theorem (k-local Hamiltonians). - Let us consider the long-range interacting Hamiltonian $H$ of the form (A7) with the assumption (A2). For $|t| \geq 1$, this Hamiltonian $H$ satisfies the Lieb-Robinson bound as follows:

$$
\frac{\left\|\left[O_{X}(t), O_{Y}\right]\right\|}{\left\|O_{X}\right\| \cdot\left\|O_{Y}\right\|} \leq \mathcal{C}_{H}^{(k)}\left|X^{\left(\bar{v}^{(k)}|t|\right)}\right| \cdot\left|Y^{\left(\bar{v}^{(k)}|t|\right)}\right| \frac{|t|^{2 D+1}}{\left(x-\bar{v}^{(k)}|t|\right)^{\alpha}}
$$

and

$$
\frac{\left\|\left[O_{X}(t), O_{Y}\right]\right\|}{\left\|O_{X}\right\| \cdot\left\|O_{Y}\right\|} \leq \mathcal{C}_{H}^{(k)^{\prime}}\left|X^{\left(\bar{v}^{(k)}|t|\right)}\right|^{2} \frac{|t|^{D+1}}{\left(x-\bar{v}^{(k)}|t|\right)^{\alpha-D}},
$$

where $\mathcal{C}_{H}^{(k)}, \mathcal{C}_{H}^{(k)^{\prime}}$, and $\bar{v}^{(k)}$ are constants that depend only on the parameters $\left\{D, g_{0}, \alpha, k\right\}$ and a geometric constant that is determined by the lattice structure.

The first and second inequalities in the above theorem reduce to the inequalities (6) and (7) in the main part, respectively; in deriving the inequality (7), we use the discussion in Ref. [2] to relate the commutator inequality to the local approximation.

\section{APPENDIX B: SKETCH OF THE PROOF}

We herein show the essential ideas, and further details are provided in Supplemental Material [117]. For the proof, we first decompose the length scale into $\leq \ell_{t}$ and $>\ell_{t}$ for a fixed $t$ and consider the following decomposition for the total Hamiltonian: $H=H_{\leq \ell_{t}}+H_{>\ell_{t}}$, where we define $H_{\leq \ell}$ for arbitrary $\ell \in \mathbb{N}$ as the operator that includes all the interaction terms whose length scales are less than $\ell$ : $H_{\leq \ell}=\sum_{\operatorname{diam}(Z) \leq \ell} h_{Z}$. In the case where the length $\ell$ is short range or $\ell=\mathcal{O}(1)$, the Hamiltonian $H_{\leq \ell}$ provides the Lieb-Robinson bound with a finite velocity. However, we consider the case of $\ell=\ell_{t}$ with $\ell_{t}$ depending on time $t$ here. In the following computations, we choose $\ell_{t}=|t|^{\tilde{\eta}}$ with $\tilde{\eta}:=1-\{[\alpha-2 D-1] /[2(\alpha-D)]\}<1$. When the length scale $\ell$ is in the middle range between $\ell=\mathcal{O}\left(t^{0}\right)$ and $\ell=\infty$, it is no longer considered trivial if the light cone for the dynamics by $H_{\leq \ell_{t}}$ is retained.

To obtain the Lieb-Robinson bound for $H_{\leq \ell}$ with a generic $\ell$, we further decompose $H_{\leq \ell}$ as follows (Fig. 5):

$$
H_{\leq \ell}=\sum_{q=1}^{q^{*}} H_{q}, \quad H_{q}:=\sum_{\ell_{q-1} \leq \operatorname{diam}(Z)<\ell_{q}} h_{Z} .
$$

We define a set of length scales $\left\{\ell_{q}\right\}_{q=1}^{q^{*}}$ as $\ell_{q}=\ell_{q-1}^{1+\eta_{q}}$ with $\eta_{q}>0$, where $\left\{\eta_{q}\right\}_{q=2}^{q^{*}}$ is appropriately chosen such that $\left\{\ell_{q}\right\}_{q=1}^{q^{*}}$ is an integer and there exists an integer $q^{*} \in \mathbb{N}$ satisfying $\ell_{q^{*}}=\ell_{1}^{\left(1+\eta_{2}\right)\left(1+\eta_{3}\right) \ldots\left(1+\eta_{q^{*}}\right)}=\ell$. In this case, $\ell_{q}$ increases by the double exponential function with respect to $q$.

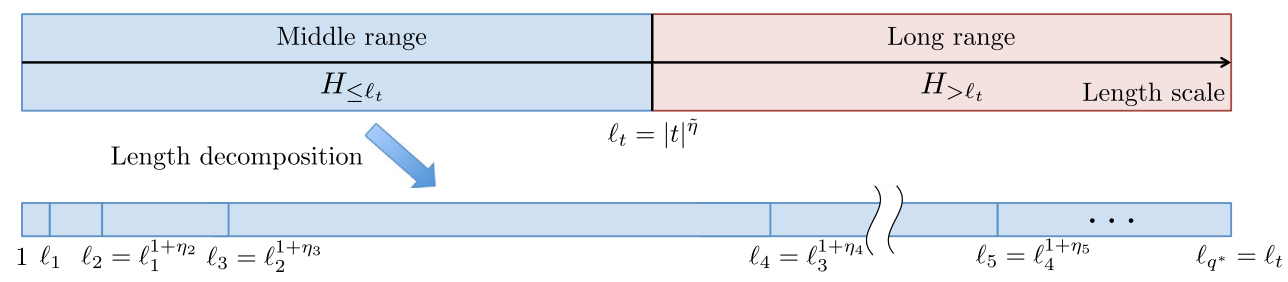

FIG. 5. Decomposition of the length scale. We first decompose the total Hamiltonian into two regimes: $H_{\leq \ell_{t}}$ and $H_{>\ell_{t}}$. The Hamiltonian $H_{\leq \ell_{t}}$ includes all the interactions up to the length scale $\ell_{t}$ that is dependent on time, and $H_{>\ell_{t}}$ includes the other interactions. To obtain the middle-range Lieb-Robinson bound, we further decompose the range $\left[1, \ell_{t}\right]$ into $q^{*}$ segments. We start from the Hamiltonian that includes only the length scale $\ell_{1}$ and iteratively consider the increasing length scales. 
In deriving the Lieb-Robinson bound for $e^{-i H_{\leq t} t}$, we iteratively take in the increasing length scales. We begin with the unitary operator $e^{-i H_{1} t}$; as long as $\ell_{1}$ is independent of $t$, the Lieb-Robinson bound for $e^{-i H_{1} t}$ is in the shorter range with a velocity of $v_{1}=\mathcal{O}(1)$. Then, using this Lieb-Robinson bound, we derive a new Lieb-Robinson bound for $e^{-i\left(H_{1}+H_{2}\right) t}=e^{-i H_{1: 2} t}$, where we define $H_{1: q}:=$ $\sum_{s=1}^{q} H_{s}$ with $1 \leq q \leq q^{*}$. This process is repeated to extend the length scales for $\ell_{1} \rightarrow \ell_{2} \rightarrow \cdots \rightarrow \ell_{q^{*}}=\ell$. In each of these steps, based on the Lieb-Robinson bound for $e^{-i H_{1: q-1} t}$, we update the Lieb-Robinson bound for $e^{-i H_{1: q} t}$.

In the first update, we start from the following decomposition of the unitary operator $e^{-i H_{1: 2} t}$ :

$$
U_{2, t}:=e^{-i\left(H_{1}+H_{2}\right) t}=e^{-i H_{1} t} \mathcal{T} e^{-\int_{0}^{t} H_{2}\left(H_{1}, \tau\right) d \tau} .
$$

The operator spreading by $e^{-i H_{1} t}$ is of the order of $\mathcal{O}\left(v_{1} t\right)$; hence, as long as $t \leq \Delta t_{2} \approx \ell_{2} / v_{1}, H_{2}\left(H_{1}, \tau\right)\left(\tau \leq \Delta t_{2}\right)$ has the same interaction length as the original, namely, $\ell_{2}$. Thus, we can obtain the Lieb-Robinson bound with a linear light cone for $\mathcal{T} e^{-\int_{0}^{\Delta t_{2}} H_{2}\left(H_{1}, \tau\right) d \tau}$. For any unspecified time $t$, we consider $U_{2, t}=U_{2, \Delta t_{2}}^{m} U_{2, \Delta t_{2}^{\prime}}$, with $t=m \Delta t_{2}+\Delta t_{2}^{\prime}$, where $\Delta t_{2} \propto \ell_{2} / v_{1}$ and $\Delta t_{2}^{\prime}<\Delta t_{2}$. By appropriately connecting all the Lieb-Robinson bounds, this process results in a Lieb-Robinson bound of the following form:

$$
\left\|\left[O_{X}\left(H_{\leq \ell_{2}}, t\right), O_{Y}\right]\right\| \lesssim\left(1+x / \ell_{2}\right)^{D-1} e^{-2\left(x-v_{2}|t|\right) / \ell_{2}}
$$

with $x=d_{X, Y}$, where the velocity $v_{2}>v_{1}$ is upper bounded using the Lieb-Robinson velocity $v_{1}$ for $e^{-i H_{1} t}$.

For general $q-1$, we define $v_{q-1}$ as the Lieb-Robinson velocity for $e^{-i H_{1: q-1} t}$ and analyze the unitary operator $U_{q, t}:=e^{-i H_{1: q} t}$, which we decompose as $U_{q, t}=$ $U_{q, \Delta t_{q}}^{m} U_{q, \Delta t_{q}^{\prime}}$ with

$$
U_{q, \Delta t_{q}}=e^{-i H_{1: q-1} \Delta t_{q}} \mathcal{T} e^{-\int_{0}^{\Delta t_{q}} H_{q}\left(H_{1: q-1}, \tau\right) d \tau}
$$

and $t=m \Delta t_{q}+\Delta t_{q}^{\prime}$; here, $\Delta t_{q} \propto \ell_{q} / v_{q-1}$ and $\Delta t_{q}^{\prime}<\Delta t_{q}$. From the choice of $\Delta t_{q}$, we can ensure that $H_{q}\left(H_{1: q-1}, \tau\right)$ has the same interaction length as that of $H_{q}$, namely, $\ell_{q}$. We then obtain the Lieb-Robinson bound for $H_{1: q}$ as follows:

$$
\left\|\left[O_{X}\left(H_{1: q}, t\right), O_{Y}\right]\right\| \lesssim\left(1+x / \ell_{q}\right)^{D-1} e^{-2\left(x-v_{q}|t|\right) / \ell_{q}},
$$

where $v_{q}$ depends on the $v_{q-1}$. Thus, we iteratively estimate the Lieb-Robinson velocity $v_{q}$ using $v_{q-1}$. Furthermore, we can derive the following recursion relation:

$$
v_{q}=v_{q-1}\left(1+\frac{c \log \left(\ell_{q}\right)}{\ell_{q-1}^{\eta}}+\frac{c^{\prime}}{\log \left(\ell_{q-1}\right)}\right),
$$

where $\eta=\sqrt{1+\{[\alpha-(2 D+1)] /[D+2]\}}-1$ and $c, c^{\prime}$ are constants. We provide the explicit form in Ref. [117]. The length scale $\ell_{q}$ is now lower bounded by a double exponential function with respect to $q$. Therefore, $\lim _{q \rightarrow \infty} v_{q}$ converges to a constant $v^{*}$, and we obtain the following "middle-range Lieb-Robinson bound" for $e^{-i H_{\leq t} t}$ :

$\left\|\left[O_{X}\left(H_{\leq \ell}, t\right), O_{Y}\right]\right\| \lesssim(1+x / \ell)^{D-1} e^{-2\left(x-v^{*}|t|\right) / \ell}$.

In this manner, we can ensure that $H_{\leq \ell}$ retains the linear light cone for $\ell \lesssim t$ from Eq. (B1), whereas no such confirmation is possible for $\ell \gtrsim t$. Thus, we consider the case of $\ell=\ell_{t}$ and decompose the total time evolution as

$$
e^{-i H t}=e^{-i H_{\leq t_{t}} t} U_{>\ell_{t}},
$$

where $U_{>\ell_{t}}:=\mathcal{T} e^{-i \int_{0}^{t} H_{>\ell_{t}}\left(H_{\leq \ell_{t}}, \tau\right) d \tau}$. In order to estimate the quasilocality of the interaction for $H_{>\ell_{t}}\left(H_{\leq \ell_{t}}, \tau\right)$, we apply the middle-range Lieb-Robinson bound (B1). Based on the quasilocality, we utilize the standard recursion approach $[14,15]$ to obtain the Lieb-Robinson bound for $U_{>\ell_{t}}$. After intricate calculations, we obtain the Lieb-Robinson bound for $U_{>\ell_{t}}$ as

$$
\left\|\left[U_{>\ell_{t}}^{\dagger} O_{X} U_{>\ell_{t}}, O_{Y}\right]\right\| \lesssim \frac{|t|^{2 D+1} \log ^{2 D}(x+1)}{\left(x-\kappa_{0} v^{*}|t|\right)^{\alpha}}
$$

where $\kappa_{0}$ is a constant. We then connect the two LiebRobinson bounds for $e^{-i H_{\leq \ell_{t} t}}$ [Eq. (B1)] and $U_{>\ell_{t}}$ [Eq. (B2)] to derive the total Lieb-Robinson bound in the main theorem.

[1] E. H. Lieb and D. W. Robinson, The Finite Group Velocity of Quantum Spin Systems, Commun. Math. Phys. 28, 251 (1972).

[2] S. Bravyi, M. B. Hastings, and F. Verstraete, LiebRobinson Bounds and the Generation of Correlations and Topological Quantum Order, Phys. Rev. Lett. 97, 050401 (2006).

[3] J. Eisert and T. J. Osborne, General Entanglement Scaling Laws from Time Evolution, Phys. Rev. Lett. 97, 150404 (2006).

[4] M. Fagotti and P. Calabrese, Evolution of Entanglement Entropy Following a Quantum Quench: Analytic Results for the XY Chain in a Transverse Magnetic Field, Phys. Rev. A 78, 010306(R) (2008).

[5] A. Hamma, F. Markopoulou, I. Prémont-Schwarz, and S. Severini, Lieb-Robinson Bounds and the Speed of Light from Topological Order, Phys. Rev. Lett. 102, 017204 (2009).

[6] L. Bonnes, F. H. L. Essler, and A. M. Läuchli, "LightCone” Dynamics after Quantum Quenches in Spin Chains, Phys. Rev. Lett. 113, 187203 (2014). 
[7] A. M. Läuchli and C. Kollath, Spreading of Correlations and Entanglement after a Quench in the One-Dimensional Bose-Hubbard Model, J. Stat. Mech. (2008) P05018.

[8] C. K. Burrell, J. Eisert, and T. J. Osborne, Information Propagation through Quantum Chains with Fluctuating Disorder, Phys. Rev. A 80, 052319 (2009).

[9] J. Jünemann, A. Cadarso, D. Pérez-García, A. Bermudez, and J. J. García-Ripoll, Lieb-Robinson Bounds for SpinBoson Lattice Models and Trapped Ions, Phys. Rev. Lett. 111, 230404 (2013).

[10] J.-S. Bernier, R. Tan, L. Bonnes, C. Guo, D. Poletti, and C. Kollath, Light-Cone and Diffusive Propagation of Correlations in a Many-Body Dissipative System, Phys. Rev. Lett. 120, 020401 (2018).

[11] D. Damanik, M. Lemm, M. Lukic, and W. Yessen, New Anomalous Lieb-Robinson Bounds in Quasiperiodic XY Chains, Phys. Rev. Lett. 113, 127202 (2014).

[12] J. Eisert and D. Gross, Supersonic Quantum Communication, Phys. Rev. Lett. 102, 240501 (2009).

[13] I. Prémont-Schwarz, A. Hamma, I. Klich, and F. Markopoulou-Kalamara, Lieb-Robinson Bounds for Commutator-Bounded Operators, Phys. Rev. A 81 , 040102(R) (2010).

[14] B. Nachtergaele and R. Sims, Lieb-Robinson Bounds and the Exponential Clustering Theorem, Commun. Math. Phys. 265, 119 (2006).

[15] M. B. Hastings and T. Koma, Spectral Gap and Exponential Decay of Correlations, Commun. Math. Phys. 265, 781 (2006).

[16] C. K. Burrell and T. J. Osborne, Bounds on the Speed of Information Propagation in Disordered Quantum Spin Chains, Phys. Rev. Lett. 99, 167201 (2007).

[17] B. Nachtergaele and R. Sims, Lieb-Robinson Bounds in Quantum Many-Body Physics, Contemp. Math. 529, 141 (2010).

[18] D. Poulin, Lieb-Robinson Bound and Locality for General Markovian Quantum Dynamics, Phys. Rev. Lett. 104, 190401 (2010).

[19] N. Schuch, S. K. Harrison, T. J. Osborne, and J. Eisert, Information Propagation for Interacting-Particle Systems, Phys. Rev. A 84, 032309 (2011).

[20] I. Prémont-Schwarz and J. Hnybida, Lieb-Robinson Bounds on the Speed of Information Propagation, Phys. Rev. A 81, 062107 (2010).

[21] M. B. Hastings, Lieb-Schultz-Mattis in Higher Dimensions, Phys. Rev. B 69, 104431 (2004).

[22] M. B. Hastings and Xiao-Gang Wen, Quasiadiabatic Continuation of Quantum States: The Stability of Topological Ground-State Degeneracy and Emergent Gauge Invariance, Phys. Rev. B 72, 045141 (2005).

[23] M. B. Hastings, An Area Law for One-Dimensional Quantum Systems, J. Stat. Mech. (2007) P08024.

[24] L. Masanes, Area Law for the Entropy of Low-Energy States, Phys. Rev. A 80, 052104 (2009).

[25] F. G. S. L. Brandão, T. S. Cubitt, A. Lucia, S. Michalakis, and D. Perez-Garcia, Area Law for Fixed Points of Rapidly Mixing Dissipative Quantum Systems, J. Math. Phys. (N.Y.) 56, 102202 (2015).
[26] K. Van Acoleyen, M. Mariën, and F. Verstraete, Entanglement Rates and Area Laws, Phys. Rev. Lett. 111, 170501 (2013).

[27] M. P. Müller, E. Adlam, L. Masanes, and N. Wiebe, Thermalization and Canonical Typicality in TranslationInvariant Quantum Lattice Systems, Commun. Math. Phys. 340, 499 (2015).

[28] L. Aolita, F. de Melo, and L. Davidovich, Open-System Dynamics of Entanglement: A Key Issues Review, Rep. Prog. Phys. 78, 042001 (2015).

[29] C. Gogolin and J. Eisert, Equilibration, Thermalisation, and the Emergence of Statistical Mechanics in Closed Quantum Systems, Rep. Prog. Phys. 79, 056001 (2016).

[30] E. Iyoda, K. Kaneko, and T. Sagawa, Fluctuation Theorem for Many-Body Pure Quantum States, Phys. Rev. Lett. 119, 100601 (2017).

[31] M. B. Hastings and S. Michalakis, Quantization of Hall Conductance for Interacting Electrons on a Torus, Commun. Math. Phys. 334, 433 (2015).

[32] M. B. Hastings, Quasi-adiabatic Continuation for Disordered Systems: Applications to Correlations, LiebSchultz-Mattis, and Hall Conductance, arXiv:1001.5280.

[33] S. Bravyi, M. B. Hastings, and S. Michalakis, Topological Quantum Order: Stability under Local Perturbations, J. Math. Phys. (N.Y.) 51, 093512 (2010).

[34] S. Bravyi and M. B. Hastings, A Short Proof of Stability of Topological Order under Local Perturbations, Commun. Math. Phys. 307, 609 (2011).

[35] S. Michalakis and J. P. Zwolak, Stability of FrustrationFree Hamiltonians, Commun. Math. Phys. 322, 277 (2013).

[36] M. B. Hastings, Locality in Quantum and Markov Dynamics on Lattices and Networks, Phys. Rev. Lett. 93, 140402 (2004).

[37] S. Hernández-Santana, C. Gogolin, J. I. Cirac, and A. Acín, Correlation Decay in Fermionic Lattice Systems with Power-Law Interactions at Nonzero Temperature, Phys. Rev. Lett. 119, 110601 (2017).

[38] M. B. Hastings, Decay of Correlations in Fermi Systems at Nonzero Temperature, Phys. Rev. Lett. 93, 126402 (2004).

[39] T. Kuwahara, T. Mori, and K. Saito, Floquet-Magnus Theory and Generic Transient Dynamics in Periodically Driven Many-Body Quantum Systems, Ann. Phys. (Amsterdam) 367, 96 (2016).

[40] F. Machado, D. V. Else, G. D. Kahanamoku-Meyer, C. Nayak, and N. Y. Yao, Long-Range Prethermal Phases of Nonequilibrium Matter, Phys. Rev. X 10, 011043 (2020).

[41] T. J. Osborne, Hamiltonian Complexity, Rep. Prog. Phys. 75, 022001 (2012).

[42] T. J. Osborne, Efficient Approximation of the Dynamics of One-Dimensional Quantum Spin Systems, Phys. Rev. Lett. 97, 157202 (2006).

[43] T. J. Osborne, Simulating Adiabatic Evolution of Gapped Spin Systems, Phys. Rev. A 75, 032321 (2007).

[44] S. Bravyi, D. P. DiVincenzo, D. Loss, and B. M. Terhal, Quantum Simulation of Many-Body Hamiltonians Using Perturbation Theory with Bounded-Strength Interactions, Phys. Rev. Lett. 101, 070503 (2008). 
[45] M. B. Hastings, Quantum Belief Propagation: An Algorithm for Thermal Quantum Systems, Phys. Rev. B 76, 201102(R) (2007).

[46] M. B. Hastings, Light-Cone Matrix Product, J. Math. Phys. (N. Y.) 50, 095207 (2009).

[47] M. B. Hastings, Observations Outside the Light Cone: Algorithms for Nonequilibrium and Thermal States, Phys. Rev. B 77, 144302 (2008).

[48] J. Haah, M. Hastings, R. Kothari, and G. H. Low, Quantum Algorithm for Simulating Real Time Evolution of Lattice Hamiltonians, in Proceedings of the 59th Annual Symposium on Foundations of Computer Science (FOCS) (IEEE, New York, 2018), pp. 350-360.

[49] M. C. Tran, A. Y. Guo, Y. Su, J. R. Garrison, Z. Eldredge, M. Foss-Feig, A. M. Childs, and A. V. Gorshkov, Locality and Digital Quantum Simulation of Power-Law Interactions, Phys. Rev. X 9, 031006 (2019).

[50] W. W. Ho, C. Jonay, and T. H. Hsieh, Ultrafast Variational Simulation of Nontrivial Quantum States with Long-Range Interactions, Phys. Rev. A 99, 052332 (2019).

[51] D. A. Roberts and B. Swingle, Lieb-Robinson Bound and the Butterfly Effect in Quantum Field Theories, Phys. Rev. Lett. 117, 091602 (2016).

[52] J. Maldacena, S. H. Shenker, and D. Stanford, A Bound on Chaos, J. High Energy Phys. 08 (2016) 106.

[53] Y. Gu, X.-L. Qi, and D. Stanford, Local Criticality, Diffusion and Chaos in Generalized Sachdev-Ye-Kitaev Models, J. High Energy Phys. 05 (2017) 125.

[54] X. Chen and T. Zhou, Quantum Chaos Dynamics in LongRange Power Law Interaction Systems, Phys. Rev. B 100, 064305 (2019).

[55] I. Kukuljan, S. Grozdanov, and T. Prosen, Weak Quantum Chaos, Phys. Rev. B 96, 060301(R) (2017).

[56] A. Nahum, J. Ruhman, S. Vijay, and J. Haah, Quantum Entanglement Growth under Random Unitary Dynamics, Phys. Rev. X 7, 031016 (2017).

[57] V. Khemani, D. A. Huse, and A. Nahum, VelocityDependent Lyapunov Exponents in Many-Body Quantum, Semiclassical, and Classical Chaos, Phys. Rev. B 98, 144304 (2018).

[58] Y.-L. Zhang, Y. Huang, and X. Chen, Information Scrambling in Chaotic Systems with Dissipation, Phys. Rev. B 99, 014303 (2019).

[59] D. J. Luitz and Y. B. Lev, Information Propagation in Isolated Quantum Systems, Phys. Rev. B 96, 020406(R) (2017).

[60] J. Li, R. Fan, H. Wang, B. Ye, B. Zeng, H. Zhai, X. Peng, and J. Du, Measuring Out-of-Time-Order Correlators on a Nuclear Magnetic Resonance Quantum Simulator, Phys. Rev. X 7, 031011 (2017).

[61] S. Pappalardi, A. Russomanno, B. Žunkovič, F. Iemini, A. Silva, and R. Fazio, Scrambling and Entanglement Spreading in Long-Range Spin Chains, Phys. Rev. B 98, 134303 (2018).

[62] D. E. Parker, X. Cao, A. Avdoshkin, T. Scaffidi, and E. Altman, A Universal Operator Growth Hypothesis, Phys. Rev. X 9, 041017 (2019).

[63] T. Zhou, S. Xu, X. Chen, A. Guo, and B. Swingle, Operator Lévy Flight: Light Cones in Chaotic Long-Range Interacting Systems, Phys. Rev. Lett. 124, 180601 (2020).
[64] M. Cheneau, P. Barmettler, D. Poletti, M. Endres, P. Schauß, T. Fukuhara, C. Gross, I. Bloch, C. Kollath, and S. Kuhr, Light-Cone-Like Spreading of Correlations in a Quantum Many-Body System, Nature (London) 481, 484 (2012).

[65] T. Langen, R. Geiger, M. Kuhnert, B. Rauer, and J. Schmiedmayer, Local Emergence of Thermal Correlations in an Isolated Quantum Many-Body System, Nat. Phys. 9, 640 (2013).

[66] F. Meinert, M. J. Mark, E. Kirilov, K. Lauber, P. Weinmann, M. Gröbner, A. J. Daley, and H.-C. Nägerl, Observation of Many-Body Dynamics in Long-Range Tunneling after a Quantum Quench, Science 344, 1259 (2014).

[67] S. Hild, T. Fukuhara, P. Schauß, J. Zeiher, M. Knap, E. Demler, I. Bloch, and C. Gross, Far-from-Equilibrium Spin Transport in Heisenberg Quantum Magnets, Phys. Rev. Lett. 113, 147205 (2014).

[68] P. Richerme, Z.-X. Gong, A. Lee, C. Senko, J. Smith, M. Foss-Feig, S. Michalakis, A. V. Gorshkov, and C. Monroe, Non-local Propagation of Correlations in Quantum Systems with Long-Range Interactions, Nature (London) 511, 198 (2014).

[69] P. Jurcevic, B. P. Lanyon, P. Hauke, C. Hempel, P. Zoller, R. Blatt, and C. F. Roos, Quasiparticle Engineering and Entanglement Propagation in a Quantum Many-Body System, Nature (London) 511, 202 (2014).

[70] V. Lienhard, S. de Léséleuc, D. Barredo, T. Lahaye, A. Browaeys, M. Schuler, L.-P. Henry, and A. M. Läuchli, Observing the Space- and Time-Dependent Growth of Correlations in Dynamically Tuned Synthetic Ising Models with Antiferromagnetic Interactions, Phys. Rev. X 8, 021070 (2018).

[71] V. Bendkowsky, B. Butscher, J. Nipper, J. P. Shaffer, R. Löw, and T. Pfau, Observation of Ultralong-Range Rydberg Molecules, Nature (London) 458, 1005 (2009).

[72] I. Bloch, J. Dalibard, and W. Zwerger, Many-Body Physics with Ultracold Gases, Rev. Mod. Phys. 80, 885 (2008).

[73] M. Saffman, T. G. Walker, and K. Mølmer, Quantum Information with Rydberg Atoms, Rev. Mod. Phys. 82, 2313 (2010).

[74] B. Yan, S. A. Moses, B. Gadway, J. P. Covey, K. R. A. Hazzard, A. M. Rey, D. S. Jin, and J. Ye, Observation of Dipolar Spin-Exchange Interactions with Lattice-Confined Polar Molecules, Nature (London) 501, 521 (2013).

[75] K. Aikawa, A. Frisch, M. Mark, S. Baier, A. Rietzler, R. Grimm, and F. Ferlaino, Bose-Einstein Condensation of Erbium, Phys. Rev. Lett. 108, 210401 (2012).

[76] J. W. Britton, B. C. Sawyer, A. C. Keith, C.-C. J. Wang, J. K. Freericks, H. Uys, M. J. Biercuk, and J. J. Bollinger, Engineered Two-Dimensional Ising Interactions in a Trapped-Ion Quantum Simulator with Hundreds of Spins, Nature (London) 484, 489 (2012).

[77] R. Islam, C. Senko, W. C. Campbell, S. Korenblit, J. Smith, A. Lee, E. E. Edwards, C.-C. J. Wang, J. K. Freericks, and C. Monroe, Emergence and Frustration of Magnetism with Variable-Range Interactions in a Quantum Simulator, Science 340, 583 (2013).

[78] H. Bernien, S. Schwartz, A. Keesling, H. Levine, A. Omran, H. Pichler, S. Choi, A. S. Zibrov, M. Endres, 
M. Greiner et al., Probing Many-Body Dynamics on a 51Atom Quantum Simulator, Nature (London) 551, 579 (2017).

[79] J. Zhang, G. Pagano, P. W. Hess, A. Kyprianidis, P. Becker, H. Kaplan, A. V. Gorshkov, Z.-X. Gong, and C. Monroe, Observation of a Many-Body Dynamical Phase Transition with a 53-Qubit Quantum Simulator, Nature (London) 551, 601 (2017).

[80] B. Neyenhuis, J. Zhang, P. W. Hess, J. Smith, A. C. Lee, P. Richerme, Z.-X. Gong, A. V. Gorshkov, and C. Monroe, Observation of Prethermalization in Long-Range Interacting Spin Chains, Sci. Adv. 3, e1700672 (2017).

[81] D. Vodola, L. Lepori, E. Ercolessi, A. V. Gorshkov, and G. Pupillo, Kitaev Chains with Long-Range Pairing, Phys. Rev. Lett. 113, 156402 (2014).

[82] T. Koffel, M. Lewenstein, and L. Tagliacozzo, Entanglement Entropy for the Long-Range Ising Chain in a Transverse Field, Phys. Rev. Lett. 109, 267203 (2012).

[83] M. F. Maghrebi, Z.-X. Gong, and A. V. Gorshkov, Continuous Symmetry Breaking in 1D Long-Range Interacting Quantum Systems, Phys. Rev. Lett. 119, 023001 (2017).

[84] T. Kuwahara, I. Arad, L. Amico, and V. Vedral, Local Reversibility and Entanglement Structure of Many-Body Ground States, Quantum Sci. Technol. 2, 015005 (2017).

[85] T. Kuwahara, Asymptotic Behavior of Macroscopic Observables in Generic Spin Systems, J. Stat. Mech. (2016) 053103.

[86] T. Kuwahara and K. Saito, Gaussian Concentration Bound and Ensemble Equivalence in Generic Quantum ManyBody Systems including Long-Range Interaction, arXiv: 1906.10872.

[87] T. Kuwahara and K. Saito, Area Law of Non-critical Ground States in 1D Long-Range Interacting Systems, arXiv:1908.11547.

[88] J. Eisert, M. van den Worm, S. R. Manmana, and M. Kastner, Breakdown of Quasilocality in Long-Range Quantum Lattice Models, Phys. Rev. Lett. 111, 260401 (2013).

[89] Z.-X. Gong, M. Foss-Feig, S. Michalakis, and A. V. Gorshkov, Persistence of Locality in Systems with Power-Law Interactions, Phys. Rev. Lett. 113, 030602 (2014).

[90] D. Métivier, R. Bachelard, and M. Kastner, Spreading of Perturbations in Long-Range Interacting Classical Lattice Models, Phys. Rev. Lett. 112, 210601 (2014).

[91] P. Hauke and L. Tagliacozzo, Spread of Correlations in Long-Range Interacting Quantum Systems, Phys. Rev. Lett. 111, 207202 (2013).

[92] M. Pino, Entanglement Growth in Many-Body Localized Systems with Long-Range Interactions, Phys. Rev. B 90, 174204 (2014).

[93] M. G. Nezhadhaghighi and M. A. Rajabpour, Entanglement Dynamics in Short- and Long-Range Harmonic Oscillators, Phys. Rev. B 90, 205438 (2014).

[94] Z. Eldredge, Z.-X. Gong, J. T. Young, A. H. Moosavian, Mi. Foss-Feig, and A. V. Gorshkov, Fast Quantum State Transfer and Entanglement Renormalization Using LongRange Interactions, Phys. Rev. Lett. 119, 170503 (2017).

[95] L. Cevolani, G. Carleo, and L. Sanchez-Palencia, Spreading of Correlations in Exactly Solvable Quantum Models with Long-Range Interactions in Arbitrary Dimensions, New J. Phys. 18, 093002 (2016).

[96] L. F. Santos, F. Borgonovi, and G. L. Celardo, Cooperative Shielding in Many-Body Systems with Long-Range Interaction, Phys. Rev. Lett. 116, 250402 (2016).

[97] G. L. Celardo, R. Kaiser, and F. Borgonovi, Shielding and Localization in the Presence of Long-Range Hopping, Phys. Rev. B 94, 144206 (2016).

[98] L. Lepori, A. Trombettoni, and D. Vodola, Singular Dynamics and Emergence of Nonlocality in Long-Range Quantum Models, J. Stat. Mech. (2017) 033102.

[99] L. Cevolani, J. Despres, G. Carleo, L. Tagliacozzo, and L. Sanchez-Palencia, Universal Scaling Laws for Correlation Spreading in Quantum Systems with Short- and LongRange Interactions, Phys. Rev. B 98, 024302 (2018).

[100] R. Singh, R. Moessner, and D. Roy, Effect of Long-Range Hopping and Interactions on Entanglement Dynamics and Many-Body Localization, Phys. Rev. B 95, 094205 (2017).

[101] X. Deng, V. E. Kravtsov, G. V. Shlyapnikov, and L. Santos, Duality in Power-Law Localization in Disordered OneDimensional Systems, Phys. Rev. Lett. 120, 110602 (2018).

[102] D. J. Luitz and Y. B. Lev, Emergent Locality in Systems with Power-Law Interactions, Phys. Rev. A 99, 010105(R) (2019).

[103] B. Kloss and Y. B. Lev, Spin Transport in a Long-RangeInteracting Spin Chain, Phys. Rev. A 99, 032114 (2019).

[104] S. Tamaki and K. Saito, Energy Current Correlation in Solvable Long-Range Interacting Systems, Phys. Rev. E 101, 042118 (2020).

[105] T. Kuwahara, Exponential Bound on Information Spreading Induced by Quantum Many-Body Dynamics with Long-Range Interactions, New J. Phys. 18, 053034 (2016).

[106] R. Sweke, J. Eisert, and M. Kastner, Lieb-Robinson Bounds for Open Quantum Systems with Long-Ranged Interactions, J. Phys. A 52, 424003 (2019).

[107] A. Y. Guo, M. C. Tran, A. M. Childs, AV. Gorshkov, and Z.-X. Gong, Signaling and Scrambling with Strongly Long-Range Interactions, arXiv:1906.02662 [Phys. Rev. A (to be published)].

[108] M. Foss-Feig, Z.-X. Gong, C. W. Clark, and A. V. Gorshkov, Nearly Linear Light Cones in Long-Range Interacting Quantum Systems, Phys. Rev. Lett. 114, 157201 (2015).

[109] T. Matsuta, T. Koma, and S. Nakamura, Improving the Lieb-Robinson Bound for Long-Range Interactions, Ann. Inst. Henri Poincaré 18, 519 (2017).

[110] D. V. Else, F. Machado, C. Nayak, and N. Y. Yao, Improved Lieb-Robinson Bound for Many-Body Hamiltonians with Power-Law Interactions, Phys. Rev. A 101, 022333 (2020).

[111] M. C. Tran, A. Ehrenberg, A. Y. Guo, P. Titum, D. A. Abanin, and A. V. Gorshkov, Locality and Heating in Periodically Driven, Power-Law-Interacting Systems, Phys. Rev. A 100, 052103 (2019).

[112] C.-F. Chen and A. Lucas, Finite Speed of Quantum Scrambling with Long Range Interactions, Phys. Rev. Lett. 123, 250605 (2019).

[113] T. Dauxois, S. Ruffo, E. Arimondo, and M. Wilkens, Dynamics and Thermodynamics of Systems with LongRange Interactions: An Introduction, in Dynamics and 
Thermodynamics of Systems with Long-Range Interactions (Springer, Berlin, 2002), pp. 1-19.

[114] A. Campa, T. Dauxois, and S. Ruffo, Statistical Mechanics and Dynamics of Solvable Models with Long-Range Interactions, Phys. Rep. 480, 57 (2009).

[115] M. C. Tran, C.-F. Chen, A. Ehrenberg, A. Y. Guo, A. Deshpande, Y. Hong, Z.-X. Gong, A. V. Gorshkov, and A. Lucas, preceding paper, Hierarchy of Linear Light Cones with Long-Range Interactions, Phys. Rev. X 10, 031009 (2020).

[116] T. Barthel and M. Kliesch, Quasilocality and Efficient Simulation of Markovian Quantum Dynamics, Phys. Rev. Lett. 108, 230504 (2012).

[117] See Supplemental Material at http://link.aps.org/ supplemental/10.1103/PhysRevX.10.031010 for the full proof of the main theorem. 